\title{
High Mycoplasma pneumoniae loads and persistent long-term Mycoplasma pneumoniae DNA in lower airway associated with severity of pediatric Mycoplasma pneumoniae pneumonia
}

Jinrong Liu' ${ }^{1 \dagger}$, Fei Zhao ${ }^{2 \dagger}$, Jie Lư ${ }^{3,4}$, Hui Xu' ${ }^{1}$, Hui Liu' ${ }^{1}$, Xiaolei Tang ${ }^{1}$, Haiming Yang ${ }^{1}$, Jianzhong Zhang ${ }^{2}$ and Shunying Zhao ${ }^{1 *}$

\begin{abstract}
Background: An increased number of refractory mycoplasma pneumoniae (MP) pneumonia (MPP) cases have been reported. However the duration of MP infection in lower airway and the course of anti-MP treatment remains unclear.

Methods: We retrospectively reviewed the medical records of 94 MPP children. Patients were classified into two groups. The long-term group (Group LT) was defined as bronchoalveolar lavage fluid (BALF) remained MP-positive by PCR after 30 days of the disease course. The non-long-term group (Group NLT) was defined as BALF became MP-negative by PCR within 30 days of disease and patients who only needed one bronchoscopy lavage therapy. MP loads, clinical outcomes were analyzed along with other clinical measurements.

Results: The average levels of inflammatory markers such as $C$ reactive protein and lactate dehydrogenase in Group LT were significantly higher than those in Group NLT. Airway and lung damage in Group LT were more severe than Group NLT. 28 patients developed necrotizing pneumonia and 8 patients developed pulmonary embolism in Group LT. Mean maximum MP loads in BALF were $10^{7.46 \pm 0.93}$ and $10^{4.86 \pm 0.93}$ in Groups LT and NLT, respectively. There was persistent MP DNA in Group LT, even lasted for 120 days. One severe MPP patient in Group LT had MP-associated bloodstream infection. After 3 months of follow-up, chest imaging revealed incomplete absorption of pulmonary consolidation in 33 patients of Group LT [including 13 airway obliterans (AO) patients] and in 7 patients of Group NLT (including 2 AO patients).
\end{abstract}

Conclusion: MP loads of BALF were associated with the subsequent duration of MP DNA in lower airway. High MP loads and persistent long-term MP DNA in lower airway were associated with severity of pediatric MPP.

Keywords: Refractory, Mycoplasma pneumoniae, Lower airway, Loads, Duration

\footnotetext{
* Correspondence: zhaoshunying2001@126.com

†Jinrong Liu and Fei Zhao contributed equally to this work.

'Department of Respiratory Medicine II, Beijing Children's Hospital, Capital

Medical University, National Center for Children's Health, No. 56, Nanlishi

Road, Xicheng District, Beijing 100045, China

Full list of author information is available at the end of the article
}

(c) The Author(s). 2019 Open Access This article is distributed under the terms of the Creative Commons Attribution 4.0 International License (http://creativecommons.org/licenses/by/4.0/), which permits unrestricted use, distribution, and reproduction in any medium, provided you give appropriate credit to the original author(s) and the source, provide a link to the Creative Commons license, and indicate if changes were made. The Creative Commons Public Domain Dedication waiver (http://creativecommons.org/publicdomain/zero/1.0/) applies to the data made available in this article, unless otherwise stated. 


\section{Background}

An increased number of refractory, severe, and even fatal Mycoplasma pneumoniae (MP) pneumonia (MPP) cases have been reported in recent year $s[1-3]$. Although the patient's body temperature can return to normal after treatment, airway and pulmonary lesions are still severe in some refractory MPP (RMPP) patients. Furthermore, some RMPP patients have sequelae, mainly atelectasis, due to airway obliterans (AO )[4].

Cell-mediated immunity and cytokine responses against the pathogens, MP resistance to macrolides and airway hypersecretion have been reported in MPP $[5,6]$. However some patients received timely treatment with sensitive antibiotics such as moxifloxacin, corticosteroid, and bronchoscopy lavage therapy (BLT), AO was still unavoidable. We observed airway hypersecretion and subsequent airway damage was severe in some children with RMPP, who required several treatments with BLT. Therefore, many factors of RMPP remain unclear, such as why airway damage persists, how long it takes to clear MP, and the course of anti-MP treatment. Elucidating these issues is important to help guide treatment strategies and further understand the underlying mechanisms of pediatric RMPP.

Here, we retrospectively analyzed MP loads in 94 MPP patients, including 90 RMPP patients. Fluorescence quantitative PCR (FQ-PCR) for MP gene detection was applied in all patients. Our studies suggested that MP could persist in the lower airway for up to 4 months, which is consistent with the severity of airway and pulmonary damage. In addition, MP culture was performed in 15 bronchoalveolar lavage fluid (BALF, MP loads $>10^{5}$ copies $/ \mathrm{mL}$ ) samples after 30 days of the disease course; 12 of 15 samples were positive, suggesting active MP infection. Moreover, MP was detected by FQPCR in the peripheral blood of one patient with severe $\mathrm{MPP}$, which suggested a rare case of MP-associated bloodstream infection. To our knowledge, this is the first report of persistent MP infection and difficult MP clearance in the lower airway of human.

\section{Methods}

\section{Study population}

We retrospectively reviewed the medical records of 94 previously healthy MPP patients who were admitted in the Department of Respiratory Medicine II at Beijing Children's Hospital affiliated to Capital Medical University, National Center for Children's Health, between January and December 2017. The study was approved by the Ethics Committee of Beijing Children's Hospital (No. 2017-23), and informed written consent was obtained from guardian of all the patients at the beginning of admission to our department. Patients with respiratory virus including adenovirus infection were excluded from current study.
In general, RMPP patients received treatment with azithromycin for 3-4 courses (nearly 1 month). In addition, it has been reported persistent MP infection of NHBE might last for up to 4 week s[7]. Therefore, patients were classified into two groups in this study. The long-term group (Group LT) was defined as BALF remained MP-positive by FQPCR after 30 days of the disease course. The non-long-term group (Group NLT) was defined as BALF became MPnegative by PCR within 30 days of disease and patients who only needed one BLT assessed by two clinicians.

The clinical and demographic data were collected and recorded for each patient. Variables included age, gender, disease course before first admission to our department, and inflammatory markers [the maximal white blood cells (WBC), C reactive protein (CRP, normal range $<8 \mathrm{mg} / \mathrm{L}$ ), lactate dehydrogenase $(\mathrm{LDH})$, normal range $<240 \mathrm{IU} / \mathrm{L}]$ within 15 days of disease course. Additionally the MP loads, findings of bronchoscopy and chest imaging, and treatment with anti-MP antibiotics and corticosteroid were recorded.

\section{Definitions}

RMPP was diagnosed according to the following criteria: (1) patients with MPP had persistent fever and deterioration of clinical and radiological findings after 7 days of disease course; (2) patients were treated with macrolides for 7 days or more $[6,8]$.

The diagnosis of $\mathrm{AO}$ was based on the obliteration of the lumen of bronchi, bronchial branches, bronchial segments, or bronchial subsegments under bronchoscopy (Fig. 1a )[6]. The imaging findings of AO were atelectasis.

\section{Fiberoptic bronchoscopy and BALF collection}

Fiberoptic bronchoscopy and BALF Collection were performed as previously reported [6].

\section{FQ PCR}

FQ PCR was performed as previously reported [9]. The standard curves for each MP Quantitative was determined by analyzing serial 10-fold dilutions of stand red MP DNA (M129 strain, 1 copy $/ \mu$ l to $10^{8}$ copies $/ \mu \mathrm{l}$ ). Each DNA of specimens and stand red was assayed in triplicate.

\section{MP genotyping and antimicrobial susceptibility testing}

MP genotyping was performed by real-time PCR assay [10]. Minimum inhibitory concentrations (MICs) against 4 antibiotics (erythromycin, azithromycin, levofloxacin, tetracycline) were determined using SP4 broth (Remel) based on the micro-dilution methods (CSLI M43-A).

\section{MP culture of BALF samples}

BALF was cultured in Mycoplasma selective liquid media (OXOID) at $37^{\circ} \mathrm{C}$. When the color of the media changed from red to yellow, approximately $0.1 \mathrm{ml}$ of the suspension 

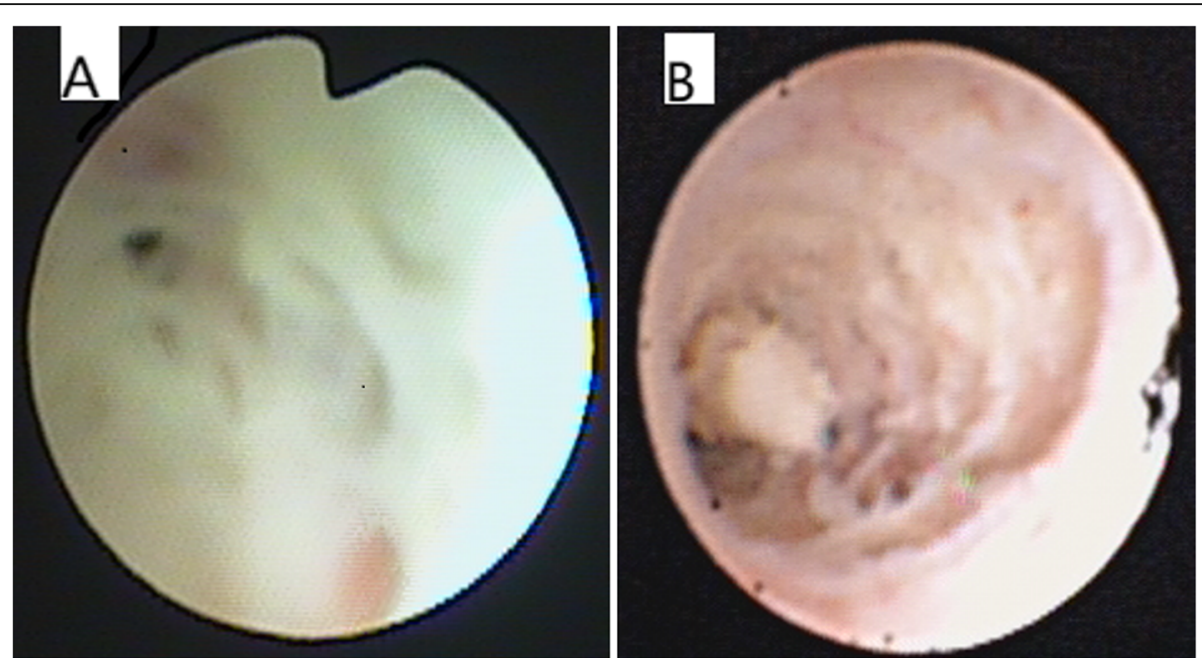

Fig. 1 a: Fiberoptic bronchoscopy revealed $A O$ (the obliteration of the lumen of bronchial branches, bronchial segments, or bronchial subsegments) in late stage of disease in RMPP patients. The rate of AO was $26 \%(13 / 50)$ and $6.8 \%$ (3/44) in Groups LT and NLT, respectively. b: Fiberoptic bronchoscopy revealed mucous plug at the early stage of disease in RMPP patients. The rate of mucus plug was $82 \%(41 / 50)$ and $6.8 \%$ (3/44) in Groups LT and NLT, respectively

was transferred onto agar to subculture and purify the bacteria using the filtration-cloning technique for MP clinical isolates.

\section{Statistical analyses}

The statistical analysis was performed in the SPSS version 17.0 (SPSS Inc., Chicago, Illinois, USA). All value data presented were expressed as mean \pm standard deviation (SD), and student's t-test was utilized for the comparison of these data between Group LT and Group NLT. The chisquare test was employed to compare categorical data. All statistical hypothesis tests were 2 -sided, and $P$ values < 0.05 were considered statistically significant.

\section{Results}

\section{Study population}

A total of 94 MPP patients (age range: 3 years 4 months13 years 7 months) were enrolled in this study. The cohort was $50 \%$ male $(n=47)$. All patients in Group LT and 40 patients in Group NLT were finally diagnosed with RMPP. There was no significant difference in gender and age between Groups LT and NLT $(P>0.05)$ (Table 1).

\section{Clinical characteristics}

The average duration of disease before hospitalization was $17.40 \pm 13.33$ days and $9.27 \pm 3.79$ days in Groups LT and NLT, respectively $(P<0.001)$.

In Group LT, most patients resided from all over the country and 12 patients had type I respiratory failure, which suggested patients in this group were severe and difficult to treat. Mean WBC count, CRP level, and LDH concentration were $11.85 \pm 4.61 \times 10^{9} / \mathrm{L}, 104.96 \pm 54.20$ $\mathrm{mg} / \mathrm{L}$, and $681.00 \pm 298.24 \mathrm{IU} / \mathrm{L}$, respectively.
In Group NLT, mean WBC count, CRP level, and LDH concentration were $8.47 \pm 2.48 \times 10^{9} / \mathrm{L}, 30.32 \pm$ $20.64 \mathrm{mg} / \mathrm{L}$, and $330.11 \pm 81.37 \mathrm{IU} / \mathrm{L}$, respectively.

A clear statistical difference was observed in inflammatory markers (WBC, CRP, LDH) between the two groups $(P<0.001)$ (Table 1).

\section{Bronchoscopy findings}

In Group LT, bronchoscopy carried out at the early stage of disease revealed mucus plug (Fig. 1b) and airway mucous necrosis (Fig.2) in 41 (including plastic bronchitis in 6 patients) and 29 patients, respectively. In the late stage of disease, 13 patients had stenosis and 13 patients had AO.

In Group NLT, three patients had a mucus plug and three patients had mucous necrosis in the early stage of disease. In the late stage of disease, bronchoscopy revealed stenosis and $\mathrm{AO}$ in three and two patients, respectively.

A clear statistical difference was observed in airway damage between the two groups $(P<0.05)$ (Table 1$)$.

\section{Chest imaging findings}

In Group LT, chest imaging at the early stage of disease revealed consolidation with high density (2/3-1 pulmonary lobe in 4 patients; $\geq 1$ lobe in 46 patients) in 50 patients and pleural effusion in 34 patients. Additionally, chest imaging revealed pulmonary embolism in 8 patients between 11 and 29 days of disease and necrotizing pneumonia (NP) in 28 patients between 14 and 60 days of disease (Fig. 3).

In Group NLT, chest imaging revealed consolidation with high density $(<1 / 2$ pulmonary lobe in 4 patients, $1 /$ $2-2 / 3$ lobe in 24 patients, and $2 / 3-1$ lobe in 11 patients) in 39 patients and bronchiolitis in 5 patients. Only two patients also had a small amount of pleural effusion. 
Table 1 Demographic and clinical features, inflammatory markers, and the findings of bronchoscopy and chest imaging in all patients

\begin{tabular}{|c|c|c|c|}
\hline & Group LT( $n=50)$ & Group NLT $(n=44)$ & $P$ Value \\
\hline Male/female & $22 / 28$ & $25 / 19$ & $P=0.215$ \\
\hline Age (years) & $7.23 \pm 2.48$ & $7.75 \pm 2.28$ & $P=0.409$ \\
\hline Disease course before first admission (days) & $17.40 \pm 13.33$ & $9.27 \pm 3.79$ & $P<0.001$ \\
\hline Respiratory failure (n/\%) & $12 /(24 \%)$ & $0 /(0 \%)$ & $P=0.001$ \\
\hline WBC $\left(\times 10^{9} / \mathrm{L}\right)$ & $11.85 \pm 4.61$ & $8.47 \pm 2.48$ & $P<0.001$ \\
\hline CRP $(\mathrm{mg} / \mathrm{L})$ & $104.96 \pm 54.20$ & $30.32 \pm 20.64$ & $P<0.001$ \\
\hline LDH (IU/L) & $681.00 \pm 298.24$ & $330.11 \pm 81.37$ & $P<0.001$ \\
\hline \multicolumn{4}{|l|}{ Bronchoscopy findings } \\
\hline \multicolumn{4}{|l|}{ Early stage } \\
\hline Mucous plug (n/\%) & $41 /(82 \%)$ & $3 /(6.8 \%)$ & $P<0.001$ \\
\hline Mucus necrosis (n/\%) & $29 /(58 \%)$ & $3 /(6.8 \%)$ & $P<0.001$ \\
\hline \multicolumn{4}{|l|}{ Late stage } \\
\hline Stenosis (n/\%) & $13 /(26 \%)$ & $3 /(6.8 \%)$ & $P=0.014$ \\
\hline $\mathrm{AO}(\mathrm{n} / \%)$ & $13 /(26 \%)$ & $2 /(4.5 \%)$ & $P=0.005$ \\
\hline \multicolumn{4}{|l|}{ Chest imaging findings } \\
\hline Consolidation (>one pulmonary lobe) (n/\%) & 46/(92\%) & $0 /(0 \%)$ & $P<0.001$ \\
\hline Pleural effusion (n/\%) & $34 /(68 \%)$ & $2 /(4.5 \%)$ & $P<0.001$ \\
\hline Pulmonary embolism (n/\%) & $8 /(16 \%)$ & $0 /(0 \%)$ & $P<0.001$ \\
\hline$N P(n / \%)$ & 28/(56\%) & $1 /(2.3 \%)$ & $P<0.001$ \\
\hline
\end{tabular}

Short names: WBC white blood cells, $C R P C$ reactive protien, $L D H$ lactate dehydrogenase, $A O$ Airway obliterans, $N P$ necrotizing pneumonia

A clear statistical difference was observed in lung damage between the two groups $(P<0.05)$ (Table 1$)$.

\section{MP loads by PCR}

$M P$ loads in paired throat swab and BALF collected at the same time point

In Group LT, throat swabs were performed in 20 patients 22-45 days after disease; 15 patients were MP- positive (including one patient with $10^{5}$ copies $/ \mathrm{mL}$ in 45 days) and 5 patients were MP-negative. Additionally, MP loads in throat swabs were lower than those in BALF. All BALF samples were MP-positive.

In Group NLT, throat swabs were performed in 10 RMPP patients 18-22 days after disease; 3 patients were MP-positive and 7 patients were MP-negative. Five BALF samples were MP-positive.
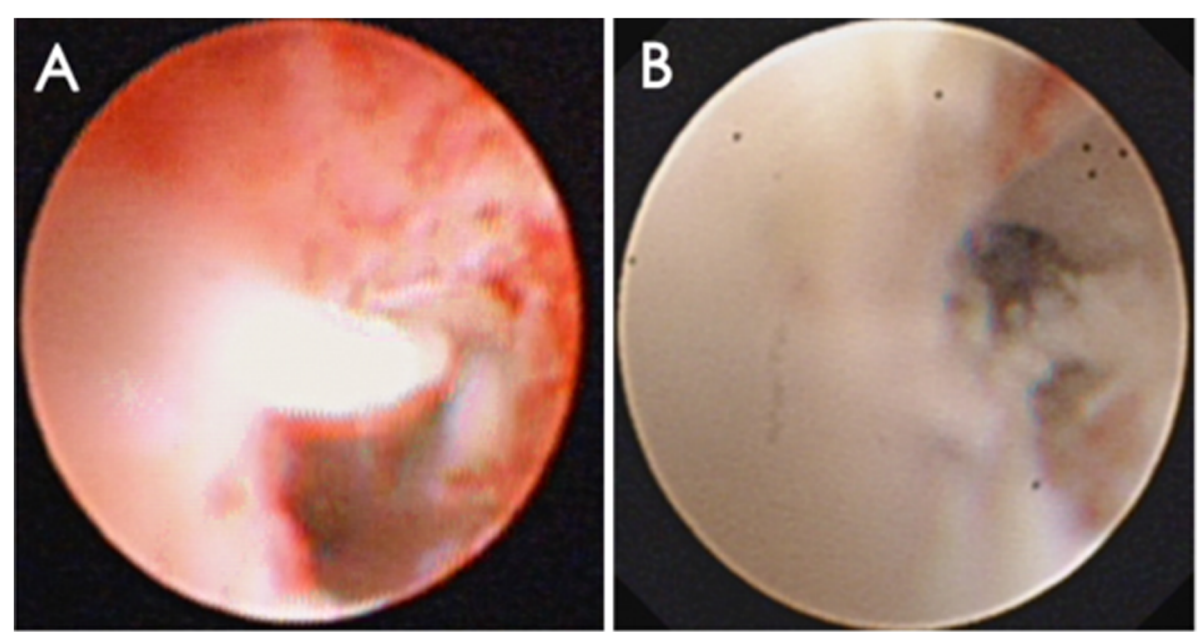

Fig. $\mathbf{2}$ a, b: Fiberoptic bronchoscopy revealed airway mucous necrosis at the early stage of disease in RMPP patients. The rate of mucus necrosis was 58\% (29/50) and 6.8\% (3/44) in Groups LT and NLT, respectively 


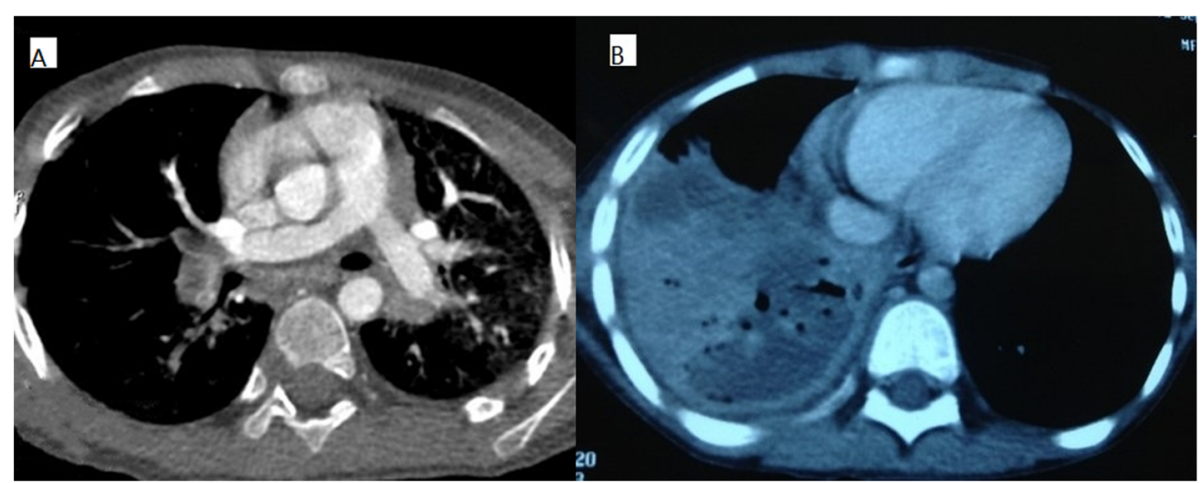

Fig. 3 a: Enhanced pulmonary CT revealed bilateral pulmonary embolism (PE) in 8 patients (16\%, 8/50) of Group LT between 11 and 29 days of disease. No one (0\%) had PE in Group NLT. b: Chest CT revealed low density lesions and multiple cavities within high density consolidation in 28 patients $(56 \%, 28 / 50)$ of Group LT between 14 and 60 days of disease. Only one patient $(2.3 \%, 1 / 44)$ had NP in Group NLT

These results suggest that patients in Group LT had longterm persistent upper airway infection and MP was cleared from the upper airway before the lower airway (Table 2).

\section{Maximum MP loads in BALF}

MP loads in BALF were classified into three groups as follows: high bacterial loads $\left(\geq 10^{7}\right.$ copies $\left./ \mathrm{mL}\right)$, moderate bacterial loads $\left(10^{6}\right.$ copies $\left./ \mathrm{mL}\right)$, and low bacterial loads $\left(<10^{6}\right.$ copies $\left./ \mathrm{mL}\right)$.

In Group LT, high, moderate, and low MP loads were found in $44(88 \%, 44 / 50), 4(8 \%, 4 / 50)$, and $2(4 \%, 2 / 50)$ patients, respectively. In Group NLT, high, moderate, and low MP loads were found in 2 (5\%,2/44), 5 (11\%, 5/ $44)$, and 37 (84\%, 37/44) patients, respectively. Mean MP loads in BALF were $10^{7.46 \pm 0.93}$ and $10^{4.86 \pm 0.93}$ in Groups LT and NLT, respectively $(P<0.001)$, which suggest MP loads in BALF are associated with the duration of MP infection.

\section{Duration of MP infection in BALF}

In Group LT, MP DNA gradually declined over time in most patients. However, in some patients, MP copy numbers initially increased and then declined. MP was detected by PCR within 31-40 days, 41-50 days, 51-60 days, and 61-90 days after disease onset in 17, 19, 9, and 4 patients, respectively. Cases 1-4 (2 NP patients and 2 AO patients) had detectable MP loads 61-90 days after disease onset. Moreover, 120 days after disease onset,

Table 2 Bacterial MP loads and duration of MP infection

\begin{tabular}{|c|c|c|}
\hline & Group LT $(n=50)$ & Group NLT $(n=44)$ \\
\hline Positive MP loads in throat swab (n/disease course/ copies/ml) & $15 /(22-45)$ days $/ 10^{3-5}$ & $3 /(18-22)$ days $/ 10^{2-3}$ \\
\hline Negative MP loads in throat swab (n/disease course) & $5 /(26-31)$ days & 7/(19-22)days \\
\hline \multicolumn{3}{|l|}{ Highest MP loads in BALF } \\
\hline High load (n/\%/copies/ml) & $44 /(88 \%) / 10^{7-8}$ & $2 /(4.54 \%) / 10^{7-8}$ \\
\hline Middle load (n/\%/copies/ml) & $4 /(8 \%) / 10^{6}$ & $5 /(11.36 \%) / 10^{6}$ \\
\hline Low load (n/\%/copies/ml) & $2 /(4 \%) / 10^{5}$ & $37 /(84.09 \%) / 10^{2-5}$ \\
\hline Highest MP loads in BALF (copies/mL) & $10^{7.46 \pm 0.93}$ & $10^{4.86 \pm 0.93}$ \\
\hline \multicolumn{3}{|l|}{ Duration of MP infection in BALF } \\
\hline Within 31-40 days (n/copies/ml) & $17 /\left(10^{2-7}\right)$ & - \\
\hline Within 41-50 days (n/copies/ml) & $19 /\left(10^{2-7}\right)$ & - \\
\hline Within 51-60 days (n/copies/ml) & $9 /\left(10^{2-7}\right)$ & - \\
\hline Within 61-90 days (n/copies/ml) & $4 /\left(10^{2-4}\right)$ & - \\
\hline Cases 1 and 2 & NP & - \\
\hline Cases 3 and 4 & $\mathrm{AO}$ & - \\
\hline 120 days (n/copies/ml) & $1 /\left(10^{3}\right)$ & - \\
\hline Case 5 & NP & - \\
\hline Duration of negative MP in BALF ( $n /$ disease course) & - & $17 /(15-30)$ days \\
\hline
\end{tabular}


MP was detected by PCR in an NP patient (Case 5, $5.98 \times 10^{3}$ copies $/ \mathrm{mL}$ ).

In Group NLT, 17 patients received more than two treatments of BLT and became MP-negative by PCR within 30 days of the disease. The remaining 27 patients received BLT once and were MP-positive by PCR.

These results demonstrate persistent long-term infection in the lower airway of patients in Group LT.

\section{MP loads in peripheral blood}

MP PCR was performed in 20 RMPP patients in Group LT within 7 days of disease. MP was detected in only one patient with severe RMPP (Case $6,3.34 \times 10^{3}$ copies $/ \mathrm{mL}$ ), which suggested a rare MP-associated bloodstream infection. This patient had type I respiratory failure and a pulmonary embolism.

\section{Detection of MP genotyping, antimicrobial susceptibility testing and MP culture in BALF}

MP genotyping, antimicrobial susceptibility testing, and MP culture was performed in 30 patients of Group LT and 11 patients of Group NLT. Genotype 2 was detected in 3 patients of Group LT and 1 patient of Group NLT. Genotype 1 was detected in the other 37 patients. Only one strain (Group NLT) was susceptible to the macrolide (genotype 2). The other 40 strains were macrolideresistant and carried the A2063G mutation. No strains with resistance to the levofloxacin and tetracycline were identified. Moreover, MP culture was performed in 15 patients of Group LT whose MP loads were $>10^{5}$ copies $/ \mathrm{mL}$ within 31-60 days after disease onset. Twelve of 15 patients were found to be MP-positive.

\section{Treatment and clinical outcomes}

All patients were treated with macrolides and BLT and followed up for at least 3 months. Four non-RMPP patients in Group NLT were not treated by glucocorticoid.

In Group LT, moxifloxacin and doxycycline were also added to the treatment course in 14 patients and 2 patients, respectively. Azithromycin, which has antiinflammatory effects, was administered for 3-6 months in 23 patients. Twenty-one patients whose CRP levels were $>100 \mathrm{mg} / \mathrm{L}$ received high-dose methylprednisolone therapy $(10-30 \mathrm{mg} / \mathrm{kg} / \mathrm{d}$ for 3 days); after 3 months, chest imaging findings were normal in only 7 patients. These seven patients had started corticosteroid treatment 7-9 days into the disease course. In Group LT, chest imaging after 3 months of treatment revealed incomplete absorption of pulmonary lesions in 33 patients, including $13 \mathrm{AO}$ patients.

In Group NLT, chest imaging after 3 months of treatment revealed incomplete absorption of pulmonary consolidation in seven patients, including two AO patients.
No obvious side effects were observed in any patients. Furthermore, there were no deaths in our study.

Taken together, these results indicate MP infection was difficult to clear, and absorption of lung lesions was very slow, especially in Group LT.

\section{Discussion}

Pediatric MPP is a significant public health problem. The clinical severity of MP infection is associated with MP loads in oropharyngeal secretion s[11, 12]. Nilsson et al. observed that the majority of patients with MPpositive throat swabs by PCR had persistent, sometimes long-term, upper airway infections (median time of carriage of MP DNA was 7 weeks after disease onset )[13]. However, to our knowledge, there is no report on the duration of MP infection in the lower airway of human. BALF is the most reliable specimen for diagnosing and assessing lower respiratory tract infection. In our study, all patients received BLT and some patients received BLT several times. We found MP was cleared in the upper airway earlier than the lower airway, and MP loads in BALF in the early stage of disease were associated with the subsequent duration of MP infection in the lower airway. Additionally, inflammatory markers (WBC, CRP, and LDH), airway and lung injury, and pulmonary complications were highly associated with $\mathrm{MP}$ loads and persistent MP DNA in BALF. Strikingly, in Group LT, all patients had persistent MP DNA even for up to 120 days, and some patients still had high MP loads 50 days after disease onset. Given that PCR cannot distinguish live bacteria from dead bacteria, we also performed MP culture for 15 patients whose MP loads were $>10^{5}$ copies $/ \mathrm{mL} ; 12$ of these patients were MPpositive, which suggested persistent long-term MP infection in the lower respiratory tract.

In our study, high MP loads and prolonged infection were mainly accompanied by airway damage, such as epithelial desquamation, necrosis, and $\mathrm{AO}$, and pulmonary damage, such as consolidation and necrosis (sometimes including recurrent low or moderate fever in few patients). These findings suggested persistent MP activation and chronic respiratory infection rather than a state of chronic MP colonization. A previous study reported that adequate antibiotic treatment does not shorten the period of persistence,[13] which was confirmed in the present study.

It has been reported that MP clearance was difficult in animal experiments or in vitro. Airway hypersecretion, epithelial desquamation, and remodeling cause $\mathrm{AO}$, which compromises further clearance in vitro,[7] which was consistent with our present study. Other studies have shown that MP is resistant to killing by neutrophils, suggesting that the bacterium might circumvent bactericidal activity of neutrophil extracellular trap s[14]. B 
cells and MP-specific antibodies are also crucial for its clearance in the lungs of mic e[15]. Interleukin (IL)-1 plays an important role in recruiting and activating lung innate immune cells that are critical for MP clearance [16]. MP respiratory tract infection in IL-12 knockout mice results in improved bacterial clearance and reduced pulmonary inflammation such as neutrophilic alveolar infiltrate and airway obstructio n[17]. Intranasal IL-12 therapy inhibits MP clearance and sustains airway obstruction in murine pneumoni a[18]. In addition, host genetic background was also found to be important for MP disease severit $y[19,20]$. However, the underlying mechanisms of persistence remain unclear.

Macrolide-resistant (MR)-MP strains are emerging and becoming increasingly common, especially in Eastern Asia, and have been reported at a rate of $>90 \%$ in Beijing from 2008 to 2012 [21]. In our present study, type 1 was the main genotype and the total MR-MP rate was high up to $97.6 \%(40 / 41)$. In addition, the MR-MP rate of genotype 1 and genotype 2 was 100\% (37/37) and 75\% (3/4) respectively, which was consistent with our previous report [22]. Macrolides are still used as first-line agents at high frequencies in countries with a high burden of MR-MP strains, such as China and Japan, mainly because of their low minimum inhibitory concentrations against the bacteria, anti-inflammatory effect and low toxicity in young children. Yang et al. reported that timely azithromycin treatment may be ineffective in treating MR-MP pneumonia in vivo, but we are unable to withhold antibiotic treatment to confirm this hypothesis due to ethical issues. However, the early use of azithromycin aids in reducing extrapulmonary complications in MPP [23]. Yoon et al. found macrolide treatment for macrolide-susceptible MPP did not contribute to significant clinical improvement compared to no antimicrobial treatment [24]. Kutty et al. reported that among MP PCR-positive children, length of stay was similar between children who did and did not receive an antibiotic with MP activity [25].

To date, no tetracycline or fluoroquinolone resistance has been reported in MP clinical isolates. Although some of our patients received timely treatment with moxifloxacin or doxycycline even for several courses, MP remained difficult to clear. Yang et al. reported anti-MP-antibiotics might have limited effects on MPP,[26] which was consistent with our study.

A previous study showed that 3-day methylprednisolone pulse therapy which was initiated 7-15 days after disease onset could be applied for RMPP treatment despite appropriate antibiotic therapy and appeared to be efficacious in 12 children [27]. However methylprednisolone pulse therapy was given 9 days after disease onset in our 14 patients in Group LT, chest imaging findings remained abnormal after 3 months of treatment. Therefore, the underlying pathogenesis of RMPP is still unclear.
Compared with C57BL/6 mice, BALB/c mice exhibited host-dependent MP infection-related airway obstruction associated with chemokine, [20] which suggested some pediatric RMPP patients were highly reactive to MP. Community-acquired respiratory distress syndrome toxin induces exfoliation of mucosal cells and ciliostasis [28]. Airway epithelial remodeling during persistent MP infection may involve epithelial-to-mesenchymal transition in a human airway epithelium model [7]. Therefore, high MP loads and epithelial desquamation, subsequent persistent MP infection, epithelial remodeling, and AO can lead to a vicious cycle of MP disease.

We found that MP may survive for a long time, especially in RMPP patients who suffer from MP associated $\mathrm{NP}$, AO, or pulmonary embolism. Furthermore, although MP-associated bloodstream infection is rare, it can occur.

Our study has several limitations. The numbers of blood samples and throat swabs were small. The reason for the small number of blood samples is that the MPnegative rate is very high, in RMPP, thus clinicians do not often request blood samples for MP detection. The number of children treated with moxifloxacin and doxycycline is small. Additionally, in some RMPP patients of Group LT, we did not store the last collected BALF sample. Therefore, the actual duration of persistence is likely underestimated.

\section{Conclusions}

Our study confirms persistent long-term MP DNA and difficult MP clearance in the lower airway of RMPP patients. Additionally, patients with MP loads $\geq 10^{7}$ copies/ $\mathrm{mL}$ in BALF are perhaps at high risk of RMPP and may be more likely to develop sequelae. Lastly, patients who have high levels of inflammatory markers, such as CRP and LDH, and high-density consolidation (especially more than one pulmonary lobe) on imaging may also have higher MP loads and subsequent persistent MP airway infection that is accompanied by NP, AO, or pulmonary embolism.

\section{Abbreviations \\ AO: Airway obliterans; BALF: Bronchoalveolar lavage fluid; BLT: Bronchoscopy lavage therapy; CRP: C reactive protein; $\mathrm{LDH}$ : Lactate dehydrogenase; LT: Long-term; MP: Mycoplasma pneumoniae; MPP: Mycoplasma pneumoniae pneumonia; MR: Macrolide-resistant; NLT: Non-long-term; RMPP: Refractory MPP; WBC: White blood cells}

\section{Acknowledgments}

We thank Christina Croney, PhD, from Liwen Bianji, Edanz Group China (www.liwenbianji.cn/ac), for editing a draft of this manuscript.

\section{Authors' contributions}

$J-R L$ and $F Z$ wrote the main manuscript text; $J L, H X, H L, X T$ and $H Y$ collected and analyzed clinical data; FZ and JZ performed MP related testing and analyzed experimental data; $J-R L, F Z, J Z$ and $S Z$ are to take responsibility for study design. All authors reviewed the manuscript. All authors read and approved the final manuscript. 


\section{Funding}

This work was supported by the National Natural Science Foundation of China (Grant No. 81772154), the Major Infectious Diseases Such as AIDS and Viral Hepatitis Prevention and Control Technology Major Projects (Grant No. 2018ZX10712-001), and Beijing Municipal and Commission Health and Family Planning (Grant No. 2015-3-076). The funding bodies had no role in the design of the study and collection, analysis, and interpretation of data and in writing the manuscript.

\section{Availability of data and materials}

The datasets generated during and/or analysed during the current study are available from the corresponding author on reasonable request.

\section{Ethics approval and consent to participate}

All procedures performed in studies involving human participants were in accordance with the ethical standards of the Ethics Committee of Beijing Children's Hospital (No. 2017-23) and with the 1964 Helsinki declaration and its later amendments or comparable ethical standards. Participant consent was written by the guardian of all the patients and informed consent forms were archived.

\section{Consent for publication}

Not applicable.

\section{Competing interests}

The authors declare that they have no competing interests.

\begin{abstract}
Author details
'Department of Respiratory Medicine II, Beijing Children's Hospital, Capital Medical University, National Center for Children's Health, No. 56, Nanlishi Road, Xicheng District, Beijing 100045, China. ${ }^{2}$ National Institute for Communicable Disease Control and Prevention, Chinese Center for Disease Control and Prevention, State Key Laboratory of Infectious Disease Prevention and Control, Beijing 102206, China. ${ }^{3}$ Key Laboratory of Major Diseases in Children, Beijing Key Laboratory for Pediatric Diseases of Otolaryngology, Head and Neck Surgery, Beijing Pediatric Research Institute, Beijing Children's Hospital, Capital Medical University, National Center for Children's Health, Beijing 100045, China. ${ }^{4}$ Biobank for Clinical Data and Samples in Pediatric, Beijing Pediatric Research Institute, Beijing Children's Hospital, Capital Medical University, National Center for Children's Health, Beijing 100045, China.
\end{abstract}

\section{Received: 26 May 2019 Accepted: 29 November 2019}

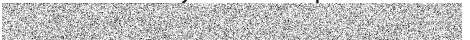

\section{References}

1. Chen L, Liu J, Zhao S, et al. Clinical features and treatment of refractory Mycoplasma pneumoniae pneumonia unresponded to conventional dose methylprednisolone in children. Zhonghua er ke za zhi = Chinese J Pediatr. 2014:52:172.

2. Miyashita N, Kawai $\mathrm{Y}$, Inamura N, et al. Setting a standard for the initiation of steroid therapy in refractory or severe mycoplasma pneumoniae pneumonia in adolescents and adults. J Infect Chemother. 2014;21:153-60.

3. Daxboeck F, Eisl B, Burghuber C, et al. Fatal mycoplasma pneumoniae pneumonia in a previously healthy 18-year-old girl. Wien Klin Wochenschr. 2007;119:379-84

4. Leong MA, Nachajon R, Ruchelli E, et al. Bronchitis obliterans due to mycoplasma pneumonia. Pediatr Pulmonol. 1997;23:375-81.

5. Wang $M$, Wang $Y$, Yan $Y$, et al. Clinical and laboratory profiles of refractory mycoplasma pneumoniae pneumonia in children. Int J Infect Dis. 2014;29:18-23.

6. Liu JR, Lu J, Dong F, et al. Low bacterial co-infection invalidates the early use of non-anti-mycoplasma pneumoniae antibiotics in pediatric refractory mycoplasma pneumoniae pneumonia patients. Front Pediatr. 2018;6:296.

7. Prince OA, Krunkosky TM, Sheppard ES, et al. Modelling persistent mycoplasma pneumoniae infection of human airway epithelium. Cell Microbiol. 2018;20:e12810.

8. Tamura A, Matsubara K, Tanaka T, et al. Methylprednisolone pulse therapy for refractory mycoplasma pneumoniae pneumonia in children. J Inf Secur. 2008;57:223-8.
9. Zhao F, Cao B, He LH, et al. Evaluation of a new real-time PCR assay for detection of mycoplasma pneumoniae in clinical specimens. Biomed Environ Sci. 2012;25:77-81.

10. Zhao F, Liu L, Tao X, He L, Meng F, Zhang J. Culture-independent detection and genotyping of mycoplasma pneumoniae in clinical specimens from Beijing. China PloS one. 2015;10(10):e0141702.

11. Dorigo-Zetsma JW, Zaat SA, Vriesema AJ, et al. Demonstration by a nested PCR for Mycoplasma pneumoniae that M pneumoniae load in the throat is higher in patients hospitalised for M pneumoniae infection than in nonhospitalised subjects. J Med Microbiol. 1999:48:1115.

12. Nilsson AC, Bjorkman P, Welinder-Olsson C, et al. Clinical severity of mycoplasma pneumoniae (MP) infection is associated with bacterial load in oropharyngeal secretions but not with MP genotype. BMC Infect Dis. 2010;10:39.

13. Nilsson AC, Björkman P, Persson $K$, et al. Polymerase chain reaction is superior to serology for the diagnosis of acute mycoplasma pneumoniae infection and reveals a high rate of persistent infection. BMC Microbiol. 2008:8:93.

14. Yamamoto T, Kida Y, Sakamoto Y, et al. Mpn491, a secreted nuclease of mycoplasma pneumoniae, plays a critical role in evading killing by neutrophil extracellular traps. Cell Microbiol. 2017;19:e12666.

15. Meyer Sauteur PM, de Groot RCA, Estevão SC, et al. The role of B cells in carriage and clearance of mycoplasma pneumoniae from the respiratory tract of mice. J Infect Dis. 2018;217:298-309.

16. Segovia JA CTWV. NLRP3 Is a Critical Regulator of Inflammation and Innate Immune Cell Response during Mycoplasma pneumoniae Infection. Infect Immun. 2017; 86. pii: e00548-17.

17. Salvatore CM, Fonseca-Aten M, Katz-Gaynor K, et al. Respiratory tract infection with mycoplasma pneumoniae in Interleukin-12 knockout mice results in improved bacterial clearance and reduced pulmonary inflammation. Infect Immun. 2006;75:236-42.

18. Salvatore CM, Fonseca-Aten M, Katz-Gaynor K, et al. Intranasal Interleukin-12 therapy inhibits mycoplasma pneumoniae clearance and sustains airway obstruction in murine pneumonia. Infect Immun. 2008:76:732-8.

19. Chu HW, Breed R, Rino JG, et al. Repeated respiratory mycoplasma pneumoniae infections in mice: effect of host genetic background. Microbes Infect. 2006;8:1764-72.

20. Fonseca-Aten M, Ríos AM, Mejías A, et al. Mycoplasma pneumoniae induces host-dependent pulmonary inflammation and airway obstruction in mice. Am J Respir Cell Mol Biol. 2005:32:201-10.

21. Zhao F, Liu G, Wu J, et al. Surveillance of macrolide-resistant mycoplasma pneumoniae in Beijing, China, from 2008 to 2012. Antimicrob Agents Chemother. 2013;57:1521-3.

22. Zhao F, Liu J, Shi W, et al. Antimicrobial susceptibility and genotyping of mycoplasma pneumoniae isolates in Beijing, China, from 2014 to 2016. Antimicrob Resist Infect Control. 2019;8:18.2

23. Yang $\mathrm{D}$, Chen $\mathrm{L}$, Chen $\mathrm{Z}$. The timing of azithromycin treatment is not associated with the clinical prognosis of childhood mycoplasma pneumoniae pneumonia in high macrolide-resistant prevalence settings. PLoS One. 2018;13:e191951.

24. Yoon IA, Hong KB, Lee $\mathrm{HJ}$, et al. Radiologic findings as a determinant and no effect of macrolide resistance on clinical course of mycoplasma pneumoniae pneumonia. BMC Infect Dis. 2017;17:402

25. Kutty PK, Jain S, Taylor TH, et al. Mycoplasma pneumoniae among children hospitalized with community-acquired pneumonia. Clin Infect Dis. 2018 May 17. https://doi.org/10.1093/cid/ciy419 [Epub ahead of print].

26. Yang EA, Kang HM, Rhim JW, et al. Early Corticosteroid Therapy for Mycoplasma pneumoniae Pneumonia Irrespective of Used Antibiotics in Children. J Clin Med. 2019; 8(5). doi: https://doi.org/10.3390/jcm8050726.

27. You SY, Jwa HJ, Yang EA, et al. Effects of Methylprednisolone Pulse Therapy on RefractoryMycoplasma pneumoniae Pneumonia in Children. Allergy, Asthma Immunol Res. 2014:6:22.

28. Kannan TR, Baseman JB. ADP-ribosylating and vacuolating cytotoxin of mycoplasma pneumoniae represents unique virulence determinant among bacterial pathogens. Proc Natl Acad Sci U S A. 2006;103:6724-9.

\section{Publisher's Note}

Springer Nature remains neutral with regard to jurisdictional claims in published maps and institutional affiliations. 